\title{
Overview of SARS-CoV-2 Infection and Its Potential Reactions
}

\author{
Esmaeil Mortaz ${ }^{1,2,{ }^{*}}$ and Neda K. Dezfuli ${ }^{1,2,3}$ \\ ${ }^{1}$ Clinical Tuberculosis and Epidemiology Research Center, National Research Institute of Tuberculosis and Lung Diseases, Shahid Beheshti University of Medical Sciences, \\ Tehran, Iran \\ ${ }^{2}$ Department of Immunology, School of Medicine, Shahid Beheshti University of Medical Sciences, Tehran, Iran \\ ${ }^{3}$ Department of Immunology and Laboratory Sciences, School of Allied Medical Sciences, Dezful University Of Medical Sciences, Dezful, Iran \\ "Corresponding author: Clinical Tuberculosis and Epidemiology Research Center, National Research Institute of Tuberculosis and Lung Diseases, Shahid Beheshti University of \\ Medical Sciences, Tehran, Iran. Email: emortaz@gmail.com
}

Received 2021 May 08; Revised 2021 June 26; Accepted 2021 June 27.

\begin{abstract}
Context: The immunopathology of SARS-CoV-2 infection in COVID-19 is not well described yet, especially regarding dysregulation of the immune system. In this mini-review, current knowledge about the SARS-CoV-2 infection and immunopathogenesis of COVID-19 disease is described. We also discuss possible induced reactions against SARS-COV-2.

Evidence Acquisition: Based on the authors' experience and knowledge, the current review aimed to, firstly, discuss and overview SRAS-CoV-2 infection and reactions in the body, and, secondly, to obtain related subjects from the PubMed database.

Results and Conclusion: In most COVID-19 patients, uncontrollable cytokines secretion and mediators are major key points in the pathogenesis of the disease. Of all cytokines and mediators, serum levels of IL-6, IL-1 $\beta$, TNF- $\alpha$, IL-8, and soluble TNF- $\alpha$ receptor (sTNFR) have been reported. Lymphopenia and hypoxia, as well as the severity of the disease, can be considered as COVID-19 manifestations. High levels of intracellular NO inside of the red blood cells (RBCs) of patients drive the unexpected silent hypoxia phenotype induced ARDS importantly related to the patient's immune system dysfunction.
\end{abstract}

Keywords: COVID-19, sTNFRI, Cytokine Storm, SARS-CoV2, Hypoxia

\section{Context}

In December 2019, a novel Coronavirus (nCoV), was emerged in Wuhan province of China. Initially, experts believed that the food market of the province played a role in disease emergence, where thousands of animals and live stocks were sold. However, further analysis of the first 41 hospitalized patients provided evidence that were not in line with this hypothesis (1). Coronavirus disease 2019, known as COVID-19, caused by the SARS-CoV-2 virus, soon expanded to other countries. As of April 2021, over $150 \mathrm{M}$ subjects were infected, and 3.1 M lives were lost worldwide (1). SARS-CoV-2 is a RNA virus belonging to the coronaviruses family that contains an envelope and can replicate in the cytoplasm of infected cells. Coronaviruses are divided into four subtypes of $\alpha, \beta, \gamma$, and $\delta$, based on their evolutionary development (2-4).

According to the currently available evidence, the SARS-CoV-2 genome consists of 29,903 nucleotides, and its GenBank number is MN908947. The genome of the virus is similar to the SARS-like coronaviruses SL-CoVZC45 and SL-CoVZXC21 derived from the bat (similarity of 88.1\%), but is remarkably different from SARS-CoV ( 79\%) and MERS-
$\mathrm{CoV}(\sim 50 \%)$ (5). It is generally accepted that the innate immune system plays a key role at the beginning of viral infections. Pattern recognition receptors (PRRs) are innate immune system receptors that can recognize specific viral elements such as RNA. When a virus enters the body, cell receptors play a major role in preventing the penetration of the virus into the viral nucleic acids. In this line, it has been shown that pattern recognition receptors (PRRs), including toll-like receptor 7 (TLR7), TLR8, RIG-I (retinoic acidinducible gene I)-like receptors (RLRs), and NOD-like receptors (NLR2), recognize ssRNA of the viruses (6).

Airway epithelial cells and innate immune cells, such as alveolar and tissue macrophages, express PRRs. In addition, RLRs can detect double-stranded (ds) RNA structures (7). PRRs stimulation by ssRNAs may result in the secretion of the type-I and -III interferons (IFNs) and chemokines (7), which is the first important response of the body to viral infections (7). In most cases, COVID-19 infection causes the secretion of uncontrollable cytokines to the blood circulation, which is a major key point in the pathogenesis of the disease (8).

During the cytokine storm induced by SARS-CoV-2 infection, the level of mediators such as IL-6, IFN- $\gamma$, IL-1 $\beta$, TNF- 
$\alpha$, and IL-8 increases, which in turn causes tissue damage, mainly in the pulmonary system and other target organs $(8,9)$. TNF- $\alpha$ plays an important role in almost all acute inflammatory responses and can exacerbate the inflammation as well. IL-1 $\beta$ is another pro-inflammatory cytokine that belongs to the inflammasome signaling and may result in fever (10). To prevent this situation, for those who suffer from inflammatory disorders such as rheumatoid arthritis and cryopyrin-associated syndromes, blocking IL$1 \beta$ is recommended (11). In addition, inflammasome inhibitor components may be prescribed for COVID-19 patients (12). It is well documented that inflammatory responses can activate neutrophils and their migration to the target organs through IL-8 chemokine (13-15). Activation and migration of neutrophils in the respiratory system during COVID-19 infection is well established (16), which is caused by higher serum levels of IL-8 (17).

Antiviral therapy can relieve the symptoms and the respiratory consequences, which can be investigated using lung CT-scan and blood markers like ESR (erythrocyte sedimentation rate), CRP (C-reactive protein), LDH (lactate dehydrogenase), and CPK (creatine phosphokinase) (9).

In the following, a review of the current knowledge about COVID-19 is provided, and the reactions and mechanisms to explain some of the symptoms are discussed.

\section{Evidence Acquisition}

Based on the authors' experience and knowledge, the current review aimed to, firstly, discuss and overview SRASCoV-2 infection and reactions in the body, and, secondly, to obtain related subjects from published articles as well as the PubMed database.

Importantly, a search on related COVID-19 and immune response and SARS-CoV-2 words is provided.

\section{Results}

\subsection{SARS-CoV-2 Reactions}

\subsubsection{Immune Dysregulation}

SARS-CoV-2 causes stimulation of both innate and adaptive immune responses, which in most cases results in overactivation and dysregulation of the immune system. However, the individual response depends on the characteristics of infected persons. The immunopathology of COVID-19 and dysregulation of the immune system is not well described; nevertheless, the general response of immune cells to this viral infection is characterized by a decreased number of lymphocytes, especially CD4 T helper cells, CD8 cells, and B cells $(7,18,19)$. Recent studies reported that severe forms of the disease are associated with a low number of $\mathrm{CD} 4+$ and $\mathrm{CD} 8+\mathrm{T}$ cells, while a reduction in $\mathrm{T}$ and $\mathrm{B}$ cells in non-severe patients has also been observed (20). Besides, some recently conducted studies reported that severe forms of the disease result in a lower ratio of CD45RA+ naïve Tregs (nTregs) and a higher ratio of CD45RO+ memory Tregs (mTregs) compared to those who suffered from mild forms of the disease (21).

Moreover, an increase in the number of naïve helper T cells (CD3+ CD4+ CD45RA+) and decreased number of memory helper cells (CD3+ CD4+ CD45RO+) in severe patients has been shown (21). Moreover, non-severe patients has an improved immune cell pattern compared to new diagnosed cases (22).

\subsubsection{Happy Hypoxia}

According to clinical evaluations, those who suffer from severe COVID-19 have declined ratio of arterial oxygen partial pressure to fractional inspired oxygen $\left(\mathrm{PaO}_{2}\right.$ : $\mathrm{FiO}_{2}$ ratio), which in turn results in hypoxia and tachypnea (23). Because of its potential to activate soluble guanylate cyclase (sGC), which influences vessels' tone and blood flow intensity, nitric oxide (NO) has a critical role in the vascular system (24). Also, through the inhibition of cytochrome c oxidase, NO can control mitochondrial oxygen consumption (21). RBCs are considered as important scavengers cells that effectively clean endothelial cell-derived NO, especially via limiting NO bioavailability in systemic NO metabolism (25). In our recent study on COVID-19 patients, we demonstrated that intracellular NO resulted in increased RBC, which in turn induced a silent phenotype of hypoxia that is a unique clinical symptom (26). It is reported that the expression level of NO inside RBC is higher in COVID-19 patients than non-COVID-19 patients with hypoxemia (26). High levels of intracellular NO in RBC of COVID-19 patients cause silent hypoxia, which is not expected; however, further evaluations are needed to expand our knowledge regarding hypoxemic in COVID-19 patients using NO of donors (23).

\subsubsection{Pneumonia in COVID-19}

While the majority of COVID-19 cases are asymptomatic or show mild symptoms, in some people, COVID-19 causes more severe symptoms with organ dysfunction (21). In the first stage of the disease (one or two days after the infection), the virus may infect epithelial cells in the nasal cavity and replicates fast. After few days, the infection expands to the upper and conducting airways, and the virus is easily detectable in nasal swabs or sputum samples (25). Simultaneously, the clinical symptoms begin to emerge, which causes activation of the immune response at high levels. In response to the increased production of IFN, the infected 
epithelial cells turn into major producers of IFN- $\gamma$ and C-XC motif chemokine ligand 10 (CXCL-10). CXCL-10 is an important immune factor in SARS and influenza viral infections and is also considered a disease marker in SARS (26). For the majority of COVID-19 patients (about 80\%), COVID19 is restricted to the upper and conducting airways (27). Nevertheless, in nearly $20 \%$ of patients, the disease progresses to the lung gas exchange units and causes hypoxia and ground-glass opacification (GGO) (28). High expression of AEC2 may be the underlying cause of severe respiratory system infection (29).

It has been shown that type II epithelial cells in the lungs are more sensitive to infection with SARS-CoV-2, and these infected cells may induce apoptosis. Since these cells had an important role in surfactant secretion, surfactant production decreasing in the airway following viral destruction of pneumocytes (30). These effects induce the alveoli collapse, which subsequently leads to pneumonia and acute respiratory distress syndrome (ARDS) in severe cases (28). According to the currently available evidence, the severity of COVID-19 disease and ARDS depends on the status of the immune system and the degree of organs involvement (31). Flow cytometry analysis of blood samples taken from critically ill COVID-19 patients showed significant declines in the number of CD3+, CD4+, and CD8+ (32). This lymphopenia is likely due to high plasma inflammatory mediators, which leads to the reduced number of $\mathrm{T}$ cells' survival/proliferation that in turn causes alerted phenotype of T-cells (28). Dysregulation of T cells may increase the severity of the disease that results in poor prognosis and even death (33).

\subsubsection{Increased Serum Levels of Soluble TNF- $\alpha$ Receptor in COVID-19 Patients}

The main clinical characteristic of severe COVID-19 patients who receive mechanical ventilation is acute respiratory distress syndrome (ARDS), which possibly is related to cytokine storm (11). We determined serum TNF- $\alpha$ and soluble TNF Receptor I (sTNFRI) levels in patients with laboratory-confirmed COVID-19, non-severe, non-ICU patients, and healthy controls. The level of sTNFRI was higher in ICU patients than non-ICU patients and healthy controls. In addition, those admitted to the ICU had higher serum levels of TNF- $\alpha$ compared to non-ICU patients and healthy controls. The sTNFRI level in serum of ICU and non-ICU patients is positively correlated with age. Also, the level of sTNFRI was negatively correlated with ESR in ICU patients. According to the findings, the serum level of sTNFRI was higher in ICU patients with severe COVID-19, and it may be a biomarker to determine the severity and mortality rate of the disease (34).

\section{Conclusions}

In conclusion, the host immune system status has a crucial role against SRAS-CoV-2 infection. It has now been a year since the start of the COVID-19 pandemic, and it is likely that this novel coronavirus will establish a niche in the body and will remain for a long time. A healthy immune system is necessary to overcome the SARS-COV-2 infection. Evaluation of immune responses of those suffering from severe and mild forms of COVID-19 disease, by taking a molecular insight into mechanisms of pathogenesis of the disease, can provide valuable information. Such information can be used to develop techniques and tools to control the COVID-19 pandemic. Eventually, COVID-19 is challenging all human beings seriously; hence, its control requires long-term collaborations, particularly among scientists and academicians, with special emphasis on international partnerships.

\section{Footnotes}

Authors' Contribution: Esmaeil Mortaz wrote the article. Neda K. Dezfuli edited the article.

Conflict of Interests: The authors confirm that there are no conflicts of interests.

Funding/Support: No funding.

\section{References}

1. Worldmeter. COVID-19 Coronavirus pandemic. USA: Worldmeter; 2021. Available from: https://www.worldometers.info/coronavirus/?utm_ campaign=homeAdvegas 1 .

2. Paules CI, Marston HD, Fauci AS. Coronavirus infections-more than just the common cold. JAMA. 2020;323(8):707-8. doi: 10.1001/jama.2020.0757. [PubMed: 31971553].

3. Ksiazek TG, Erdman D, Goldsmith CS, Zaki SR, Peret T, Emery S, et al. A novel coronavirus associated with severe acute respiratory syndrome. N Engl J Med. 2003;348(20):1953-66. doi: 10.1056/NEJMoa030781. [PubMed: 12690092].

4. Alipoor SD, Mortaz E, Jamaati H, Tabarsi P, Bayram H, Varahram M, et al. COVID-19: Molecular and cellular response. Front Cell Infect Microbiol. 2021;11:563085. doi: 10.3389/fcimb.2021.563085. [PubMed: 33643932]. [PubMed Central: PMC7904902].

5. Huang C, Wang Y, Li X, Ren L, Zhao J, Hu Y, et al. Clinical features of patients infected with 2019 novel coronavirus in Wuhan, China. Lancet. 2020;395(10223):497-506. doi: 10.1016/S0140-6736(20)30183-5. [PubMed: 31986264]. [PubMed Central: PMC7159299].

6. Li G, Fan Y, Lai Y, Han T, Li Z, Zhou P, et al. Coronavirus infections and immune responses. J Med Virol. 2020;92(4):424-32. doi: 10.1002/jmv.25685. [PubMed: 31981224]. [PubMed Central: PMC7166547].

7. Mortaz E, Tabarsi P, Varahram M, Folkerts G, Adcock IM. The immune response and immunopathology of COVID-19. Front Immunol. 2020;11:2037. doi: 10.3389/fimmu.2020.02037. [PubMed: 32983152]. [PubMed Central: PMC7479965]. 
8. Iannaccone G, Scacciavillani R, Del Buono MG, Camilli M, Ronco C, Lavie CJ, et al. Weathering the cytokine storm in COVID-19: Therapeutic implications. Cardiorenal Med. 2020;10(5):277-87. doi: 10.1159/000509483. [PubMed: 32599589]. [PubMed Central: PMC7360507].

9. Mortaz E, Bassir A, Dalil Roofchayee N, Dezfuli NK, Jamaati H, Tabarsi P, et al. Serum cytokine levels of COVID-19 patients after 7 days of treatment with favipiravir or kaletra. Int Immunopharmacol. 2021;93:107407. doi: 10.1016/j.intimp.2021.107407. [PubMed: 33631512]. [PubMed Central: PMC7826095].

10. McGeough MD, Wree A, Inzaugarat ME, Haimovich A, Johnson CD, Pena CA, et al. TNF regulates transcription of NLRP3 inflammasome components and inflammatory molecules in cryopyrinopathies. J Clin Invest. 2017;127(12):4488-97. doi: 10.1172/JCI90699. [PubMed: 29130929]. [PubMed Central: PMC5707143].

11. Hojyo S, Uchida M, Tanaka K, Hasebe R, Tanaka Y, Murakami M, et al. How COVID-19 induces cytokine storm with high mortality. Inflamm Regen. 2020;40:37. doi: 10.1186/s41232-020-00146-3. [PubMed: 33014208]. [PubMed Central: PMC7527296].

12. Dezfuli NK, Adcock IM, Montazami N, Mortaz E, Velayati A. Update on immunology of COVID-19 disease and potential strategy for controlling. Tanaffos. 2020;19(4):274-90. [PubMed: 33959165]. [PubMed Central: PMC8088154].

13. Mortaz E, Rad MV, Johnson M, Raats D, Nijkamp FP, Folkerts G. Salmeterol with fluticasone enhances the suppression of IL-8 release and increases the translocation of glucocorticoid receptor by human neutrophils stimulated with cigarette smoke. J Mol Med (Berl).2008;86(9):1045-56. doi:10.1007/s00109-008-0360-0. [PubMed: 18600309]. [PubMed Central: PMC2517086].

14. Mortaz E, Lazar Z, Koenderman L, Kraneveld AD, Nijkamp FP, Folkerts G. Cigarette smoke attenuates the production of cytokines by human plasmacytoid dendritic cells and enhances the release of IL-8 in response to TLR-9 stimulation. Respir Res. 2009;10:47. doi: 10.1186/14659921-10-47. [PubMed: 19515231]. [PubMed Central: PMC2701931].

15. Sarir H, Mortaz E, Janse WT, Givi ME, Nijkamp FP, Folkerts G. IL-8 production by macrophages is synergistically enhanced when cigarette smoke is combined with TNF-alpha. Biochem Pharmacol. 2010;79(5):698-705. doi: 10.1016/j.bcp.2009.10.001. [PubMed: 19874800].

16. Borges L, Pithon-Curi TC, Curi R, Hatanaka E. COVID-19 and neutrophils: The relationship between hyperinflammation and neutrophil extracellular traps. Mediators Inflamm. 2020;2020:8829674. doi: 10.1155/2020/8829674. [PubMed: 33343232]. [PubMed Central: PMC7732408]

17. Li L, Li J, Gao M, Fan H, Wang Y, Xu X, et al. Interleukin-8 as a biomarker for disease prognosis of Coronavirus disease-2019 patients. Front Immunol. 2020;11:602395. doi: 10.3389/fimmu.2020.602395. [PubMed: 33488599]. [PubMed Central: PMC7820901].

18. Qin C, Zhou L, Hu Z, Zhang S, Yang S, Tao Y, et al. Dysregulation of immune response in patients with Coronavirus 2019 (COVID-19) in Wuhan, China. Clin Infect Dis. 2020;71(15):762-8. doi: 10.1093/cid/ciaa248. [PubMed: 32161940]. [PubMed Central: PMC7108125].

19. Rezaei M, Marjani M, Mahmoudi S, Mortaz E, Mansouri D. Dynamic changes of lymphocyte subsets in the course of COVID-19. Int Arch Allergy Immunol. 2021;182(3):254-62. doi: 10.1159/000514202. [PubMed: 33498051]. [PubMed Central: PMC7900469].

20. Liu B, Han J, Cheng X, Yu L, Zhang L, Wang W, et al. Reduced numbers of T cells and B cells correlates with persistent SARS-CoV-2 presence in non-severe COVID-19 patients. Sci Rep. 2020;10(1):17718. doi: 10.1038/s41598-020-73955-8. [PubMed: 33077873]. [PubMed Central: PMC7573596].

21. Chen G, Wu D, Guo W, Cao Y, Huang D, Wang H, et al. Clinical and immunological features of severe and moderate coronavirus disease 2019. J Clin Invest. 2020;130(5):2620-9. doi: 10.1172/JCI137244. [PubMed 32217835]. [PubMed Central: PMC7190990].

22. Liu B, Han J, Cheng X, Yu L, Zhang L, Wang W, et al. Persistent SARS $\mathrm{CoV}-2$ presence is companied with defects in adaptive immune system in non-severe COVID-19 patients. medRxiv. 2020;Preprint. doi: 10.1101/2020.03.26.20044768.

23. Wang D, Hu B, Hu C, Zhu F, Liu X, Zhang J, et al. Clinical characteristics of 138 hospitalized patients with 2019 novel coronavirusinfected pneumonia in Wuhan, China. JAMA. 2020;323(11):1061-9. doi: 10.1001/jama.2020.1585. [PubMed: 32031570]. [PubMed Central: PMC7042881].

24. Chen K, Pittman RN, Popel AS. Nitric oxide in the vasculature: Where does it come from and where does it go? A quantitative perspective. Antioxid Redox Signal. 2008;10(7):1185-98. doi: 10.1089/ars.2007.1959. [PubMed: 18331202]. [PubMed Central: PMC2932548].

25. Cameron MJ, Bermejo-Martin JF, Danesh A, Muller MP, Kelvin DJ Human immunopathogenesis of severe acute respiratory syndrome (SARS). Virus Res. 2008;133(1):13-9. doi: 10.1016/j.virusres.2007.02.014. [PubMed: 17374415]. [PubMed Central: PMC7114310].

26. Mortaz E, Malkmohammad M, Jamaati H, Naghan PA, Hashemian SM, Tabarsi P, et al. Silent hypoxia: Higher NO in red blood cells of COVID19 patients. BMC Pulm Med. 2020;20(1):269. doi: 10.1186/s12890-02001310-8. [PubMed: 33066765]. [PubMed Central: PMC7563910].

27. Mason RJ. Pathogenesis of COVID-19 from a cell biology perspective. Eur Respir J. 2020;55(4). doi: 10.1183/13993003.00607-2020. [PubMed: 32269085]. [PubMed Central: PMC7144260].

28. Oran DP, Topol EJ. Prevalence of asymptomatic SARS-CoV-2 Infection : A narrative review. Ann Intern Med. 2020;173(5):362-7. doi: 10.7326/M20-3012. [PubMed: 32491919]. [PubMed Central: PMC7281624].

29. Li G, He X, Zhang L, Ran Q, Wang J, Xiong A, et al. Assessing ACE2 expression patterns in lung tissues in the pathogenesis of COVID-19. J $A u$ toimmun. 2020;112:102463. doi: 10.1016/j.jaut.2020.102463. [PubMed: 32303424]. [PubMed Central: PMC7152872].

30. Carcaterra M, Caruso C. Alveolar epithelial cell type II as main target of SARS-CoV-2 virus and COVID-19 development via NF-Kb pathway deregulation: A physio-pathological theory. Med Hypotheses. 2021;146:110412. doi: 10.1016/j.mehy.2020.110412. [PubMed: 33308936]. [PubMed Central: PMC7681037].

31. Yuki K, Fujiogi M, Koutsogiannaki S. COVID-19 pathophysiology: A review. Clin Immunol. 2020;215:108427. doi: 10.1016/j.clim.2020.108427. [PubMed: 32325252]. [PubMed Central: PMC7169933].

32. Coperchini F, Chiovato L, Croce L, Magri F, Rotondi M. The cytokine storm in COVID-19: An overview of the involvement of the chemokine/chemokine-receptor system. Cytokine Growth Factor Rev. 2020;53:25-32. doi: 10.1016/j.cytogfr.2020.05.003. [PubMed: 32446778]. [PubMed Central: PMC7211650].

33. Alipoor SD, Jamaati H, Tabarsi P, Mortaz E. Immunopathogenesis of pneumonia in COVID-19. Tanaffos. 2020;19(2):79-82. [PubMed: 33262791]. [PubMed Central: PMC7680509].

34. Mortaz E, Tabarsi P, Jamaati H, Dalil Roofchayee N, Dezfuli NK, Hashemian SM, et al. Increased serum levels of soluble TNF-alpha receptor is associated with ICU mortality in COVID-19 patients. Front Immunol. 2021;12:592727. doi: 10.3389/fimmu.2021.592727. [PubMed: 33968010]. [PubMed Central: PMC8100036]. 